\title{
Visuomotor Adaptation in Normal Aging
}

\author{
Ethan R. Buch, ${ }^{1}$ Sereniti Young, ${ }^{1}$ and José L. Contreras-Vidal ${ }^{1,2,3}$ \\ ${ }^{1}$ Department of Kinesiology; ${ }^{2}$ Neuroscience and Cognitive Science, University of Maryland, College Park, Maryland 20742, USA
}

\begin{abstract}
Visuomotor adaptation to a gradual or sudden screen cursor rotation was investigated in healthy young and elderly subjects. Both age groups were equally divided into two subgroups; one subgroup was exposed to $11.25^{\circ}$ step increments of visual feedback rotation, every 45 trials (up to a total of $90^{\circ}$ ), whereas a second subgroup was subjected to $90^{\circ}$ rotation from the onset of exposure. Participants performed discrete, horizontal hand movements to virtual targets in four randomized directions. Targets appeared on a computer screen in front of them, and a board prevented vision of the hand at all times. Differential effects of aging on visuomotor adaptation were found, depending on the time course of the visual distortion. In both age groups, early exposure to the sudden visual feedback distortion resulted in typical spiral-like trajectories, which became straighter by late exposure. However, the final adaptation level was reduced in the aged group, although the aftereffects were similar. When subjects were exposed to the gradual distortion, no statistically significant differences in measures of adaptation with advancing age were found. In this case, both age groups appeared to adapt equally. However, after removal of the distortion, elderly subjects showed reduced aftereffects as compared with the young group. These findings suggest differential effects of aging on adaptation to gradual versus sudden visual feedback distortions, and may help to explain the conflicting results obtained in previous visuomotor adaptation studies.
\end{abstract}

One of the fundamental properties of the human brain is its ability to adapt to changing intrinsic (e.g., growth and aging) and/or extrinsic (e.g., changing environment) conditions. Sensory-motor behavior requires the transformation and integration of information from different modalities and different coordinate systems. In visually guided reaching, the location (or spatial direction) of an object with respect to the initial position of the hand needs to be transformed into motor commands that move the arm toward the target. This visuomotor transformation needs to be updated if environmental conditions change. For example, the mapping between visual space and motor commands can be altered by artificially rotating and/or scaling the visual space via manipulation of the real-time visual feedback of hand movements displayed on a computer monitor. These visuomotor manipulations are termed screen cursor (or display) rotation and screen cursor (or display) gain, respectively.

The effects of aging on adaptive visuomotor mechanisms and behavioral responses are controversial. Some studies found no age-related adaptation deficits (Canavan et al. 1990; Roller et al. 2002); whereas others suggest that aging results in slower and reduced adaptation (Etnier and Landers 1998; McNay and Willingham 1998; Fernandez-Ruiz et al. 2000; Teulings et al. 2002). In terms of aftereffects (a measure of visuomotor learning), the results are also confusing. In prism adaptation tasks, Fernandez-Ruiz et al. (2000) reported larger aftereffects in the older group;

${ }^{3}$ Corresponding author.

E-MAIL pepeum@wam.umd.edu; FAX (301) 405-5578.

Article and publication are at http://www.learnmem.org/cgi/doi/ $10.1101 / \mathrm{lm} .50303$. whereas Roller et al. (2002) did not find age differences in aftereffects. Moreover, McNay and Willingham (1998) showed that aging does not affect the aftereffects during post-exposure if participants are told of the absence of the visual distortion at the start of this phase.

It is plausible that differences in the instructions to participants, degree of visuospatial awareness of the distorted environments, cognitive status of participants, and/ or differences in the experimental paradigms or even analysis procedures may account for some of the differences summarized above. Nevertheless, the age-related decline observed in visuomotor adaptation reported in some of the studies above could be explained by reduced plasticity (Pratt et al. 1994; Mirmiran et al. 1996), increased movement variability with age (Cooke et al. 1989; Darling et al. 1989), deficits in visuospatial working memory (West 1996; Raz et al. 2000; Richardson and Vecchi 2002), and degraded cognitive/strategic processing (McNay and Willingham 1998; Fernandez-Ruiz et al. 2000; Raz et al. 2000). These possible explanations have important consequences for both determining the underlying basis for deterioration of visuomotor mechanisms in aging and for devising new intervention procedures for new skill learning in elderly populations.

Recently, several studies have shown that the time course of kinematic distortions (e.g., due to changes in display gain or display rotation) influence the level of adaptation and aftereffects in young healthy subjects. Thus, participants exposed to a gradual distortion show improved adaptation, reduced spatio-temporal movement variability, and larger aftereffects than those participants exposed to sudden (step) visuomotor distortions (Kagerer et al. 1997;

LEARNING \& MEMORY 10:55-63 @ 2003 by Cold Spring Harbor Laboratory Press ISSN1072-0502/03 \$5.00

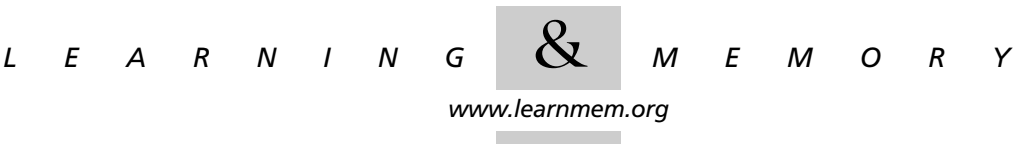


Robertson and Miall 1999; Ingram et al. 2000). Importantly, it was noted (as in the present study) that whereas participants in the step adaptation paradigm became aware of the distortion at the onset of the first exposure trial, participants in the gradual condition were not aware of the distortion until after they experienced the first post-exposure trial (in fact, a step change from the distorted to the baseline environment). This suggests that step adaptation involves learning to reduce visuospatial errors using explicit cognitive strategies, whereas gradual adaptation may engage primarily implicit learning mechanisms. Thus, as participants in the gradual paradigm are not aware of the introduction of the distortion, the use of potential cognitive strategies to solve the visuomotor discordance is minimized in this case. Finally, as gradual distortions result in smaller spatio-temporal variability than step distortions, the signal-to-noise ratios in the feedback signals used for learning are enhanced, resulting in better learning.

In summary, the visuomotor adaptation paradigm can be viewed as an active task that requires transformation, integration, modification, and storage of visuospatial and kinesthetic information. This storage and manipulation of visuospatial information, however, appears to be affected by verbal processes (e.g., oral instructions to participants), attentional processes (e.g., awareness of visual distortions), the quality of the visual feedback signals (e.g., signal to noise ratio), and the task complexity (e.g., degree of rotation). In fact, Abeele and Bock (2001) have shown that although the adaptation to small rotations can be achieved by gradually shifting the internal model of the transformation, adaptations to large rotations may require two processing stages - a polarity inversion of two axes $\left(=180^{\circ}\right)$, followed by a backward shift toward a smaller rotation angle.

In the present study, we varied the time course of visual distortions to investigate the effects of aging on the acquisition of an internal model of a novel environment (e.g., a screen cursor rotation) in both gradual and step (sudden) visual distortion regimes (Fig.

$1 \mathrm{~A}, \mathrm{~B})$. We hypothesized that gradual, as compared with sudden, cursor rotations would enhance visuomotor adaptation learning in the elderly as the extent of required adaptation at each rotation step (e.g., task complexity), the use of cognitive strategies, and the spatio-temporal movement variability are reduced in this regime. We also predicted that if aging degraded the explicit selection and implementation of cognitive strategies, elderly participants would show better learning and performance in gradual (implicit) adaptation as opposed to step (explicit) adaptation. Importantly, if the instructions to participants, the experi- mental setup, and the cognitive status of the participants were kept the same, age differences could not be attributed to these factors.

\section{RESULTS}

\section{Movement Paths}

Movement trajectories (mean \pm SD) observed during the last five trials of the pre-exposure condition, in the absence of visual feedback rotation, were similar across all subgroups (Fig. 2, Pre-exposure). Specifically, the pointing movements to the four targets during the pre-exposure condition were relatively straight for both young and elderly participants.

As expected during the initial five trials of the exposure condition, the sudden rotation had a much more pronounced effect on the movement path than the gradual rotation in both age groups. We noted that in the sudden rotation condition, subjects appeared to explore the workspace extensively, showing a combination of clockwise spiral-like trajectories as well as sudden (angle or jagged actions; see Roby-Brami and Burnod 1995) reversals in movement direction, all of which resulted in large spatial movement variability during the early exposure period, particularly in the young group (see Sudden Rotation in the Early exposure in Fig. 2). This is consistent with the large mean and SD of the initial direction of movement in early exposure trials during sudden rotation (see insets in Fig. 2). Although all participants became aware of these pronounced deviations upon performing the first trial under the $90^{\circ}$ visual rotation, debriefing by the experimenters indicated that by the end of the experiment, none of them were able to recognize the nature and/or magnitude of the distortion.

In agreement with prior reports (Kagerer et al. 1997), subjects showed straighter movement paths throughout the exposure condition in the gradual distortion condition. In
A

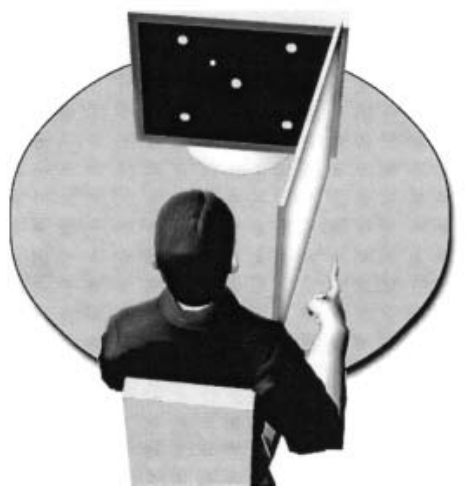

B

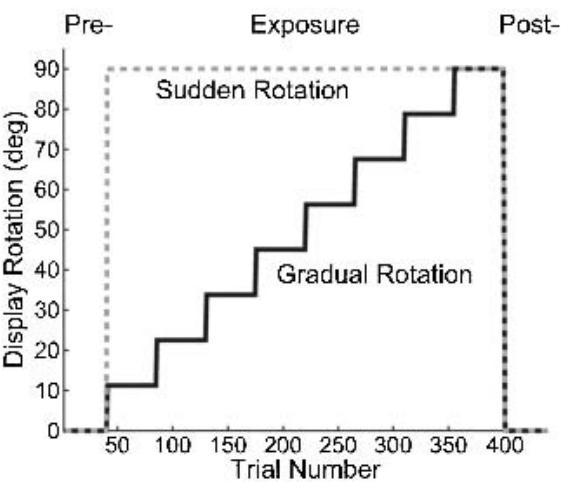

Figure 1 (A) Depiction of the experimental set-up. (B) Time-course of the sudden and gradual visual feedback distortion as a function of trial.

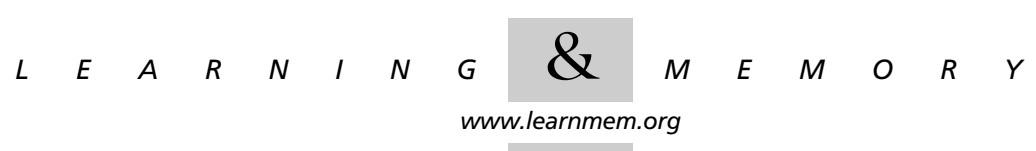




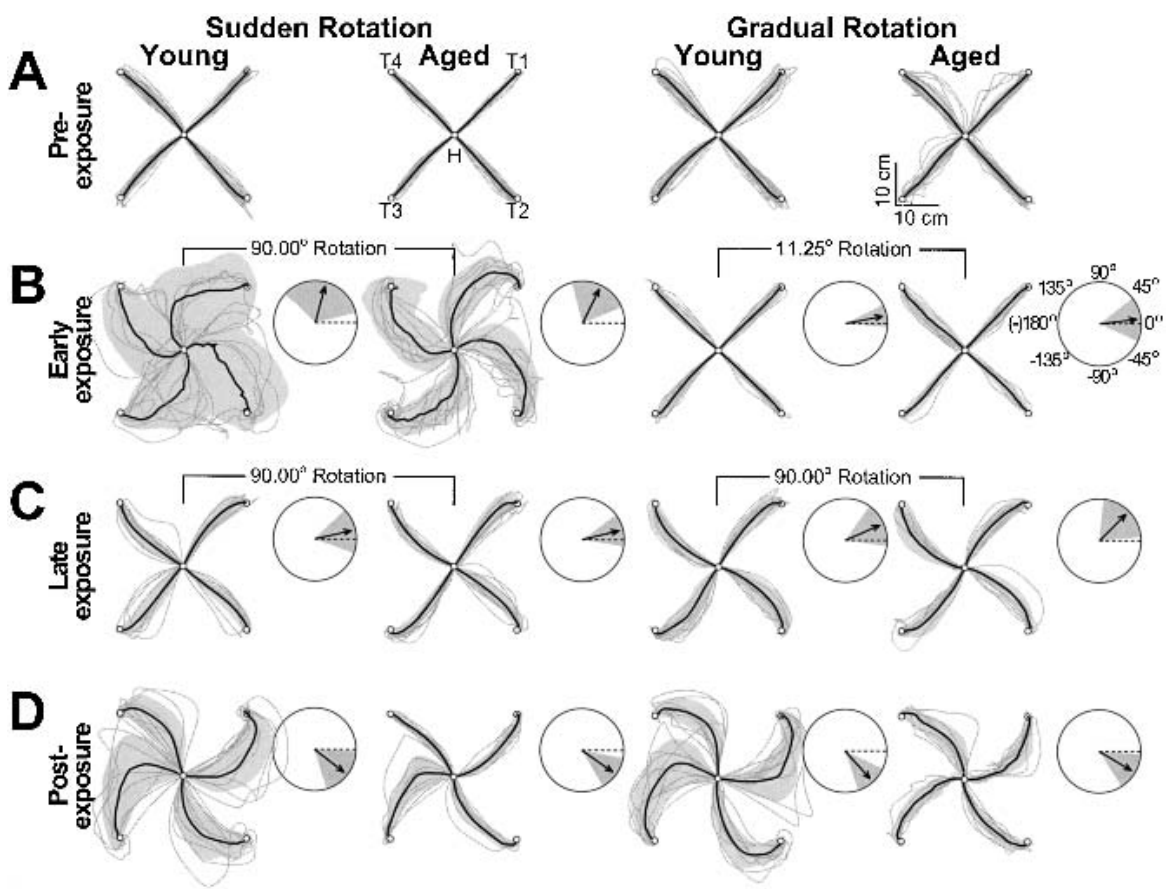

Figure 2 Screen cursor movement paths (mean $\pm S D$ ) and normalized shifts of initial direction of movement (mean $\pm \mathrm{SD}$; insets) for the young and aged groups as a function of distortion type and stage of visuomotor adaptation. (A) Pre-exposure, $(B)$ early-, and $\left(C\right.$ ) late-exposure to a $90^{\circ} \mathrm{CCW}$ screen cursor rotation, and $(D)$ post-exposure following removal of the screen cursor rotation. In these figures, the last two trials of pre- and late-exposure, and the first two trials of early and post-exposure for each subject per target direction (10 traces total per target direction for each condition) have been overlaid to show typical movement paths at different stages of learning (e.g., sharp reversals, spirals, loops, etc). (Insets) The dark vectors represent mean normalized shifts of the initial movement direction, collapsed across targets, from pre-exposure to early- $(B)$, late- $(C)$, and post-exposure trials $(D)$, respectively. The mean initial direction of movement at pre-exposure (represented as a broken dark line) is set to $0^{\circ}$.

fact, over the last five trials of exposure (Late exposure in Fig. 2), young and elderly subjects for each distortion type were able to move to the directed target relatively straight, although some shallow-arched paths and some path reversals remained. Therefore, subjects were able to adapt to the extent in which the movements were almost similar to those performed at pre-exposure. Immediately following the rotation removal (see Post exposure in Fig. 2), aftereffects were seen in both groups, suggesting that both young and elderly subjects were to some extent able to update their internal model of the transformation, and thus, their planned, initial movement direction. Visual inspection of the initial direction of movement at post-exposure (see insets in Fig. 2D) shows that the initial movement direction at post-exposure was comparable for both age groups for the sudden condition only. Moreover, a larger directional aftereffect was observed in the gradual condition for the young group compared with the elderly group. Thus, the elderly did not adapt their internal model of the initial direction of movement in the gradual distortion to the same extent as the young controls.

\section{Effects of Distortion Type (Sudden vs. Gradual)}

To investigate the effects of sudden versus gradual distortions on visuomotor adaptation, measures related to both planning (IDE) and execution (RMSE) components of the movement were analyzed.

Figure 3 compares the effects of age group (Young; Elderly) and distortion type (Gradual; Sudden) on the time courses for the standardized IDE and RMSE scores as a function of trial block. From the nonlinear fitting procedure, it was found that the error scores were better fitted with double exponentials during exposure trials and with single exponentials functions during post-exposure trials. As expected, during the initial trials of the exposure condition, the magnitude of the standardized IDE and RMSE scores in the sudden cursor rotation was much larger than that in the gradual distortion type. Both age groups responded similarly to the sudden distortion, as a Kruskal-Wallis test comparing the two age groups revealed that the differences in the mean standardized IDE and RMSE initial response (e.g., in early-exposure trials) to the step distortion did not reach statistical significance (both $P>0.05$ ).

The small rotation steps in the Gradual distortion condition resulted in reduced standardized IDE and RMSE scores throughout the exposure phase. As expected, there were not significant age differences in the early response to the distortion as assessed by the Kruskal-Wallis test $(P>$ 0.05). Note, however, that the standardized IDE and RMSE scores slowly increased with the magnitude of the rotation and eventually leveled off during the gradual distortion. This was true for both age groups. Repeated-measures MANOVA showed a main effect of rotation block (Wilk's $\lambda$; $\mathrm{F}[14,110]=10.433 ; P<0.001)$, but no main effect of age or age by rotation-block interaction (both $P>0.05$ ).

\section{Final Level of Adaptation}

To assess differences in the final level of adaptation as a function of age and distortion type, we used the KruskalWallis test to compare the means of the trial-blocks during the adaptation to the $90^{\circ}$ rotation (e.g., late-exposure). As reflected in Figure 3, a significant age effect during Step

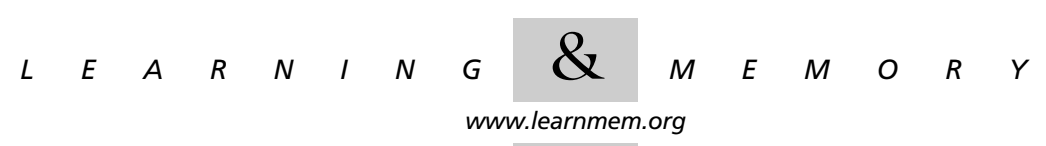



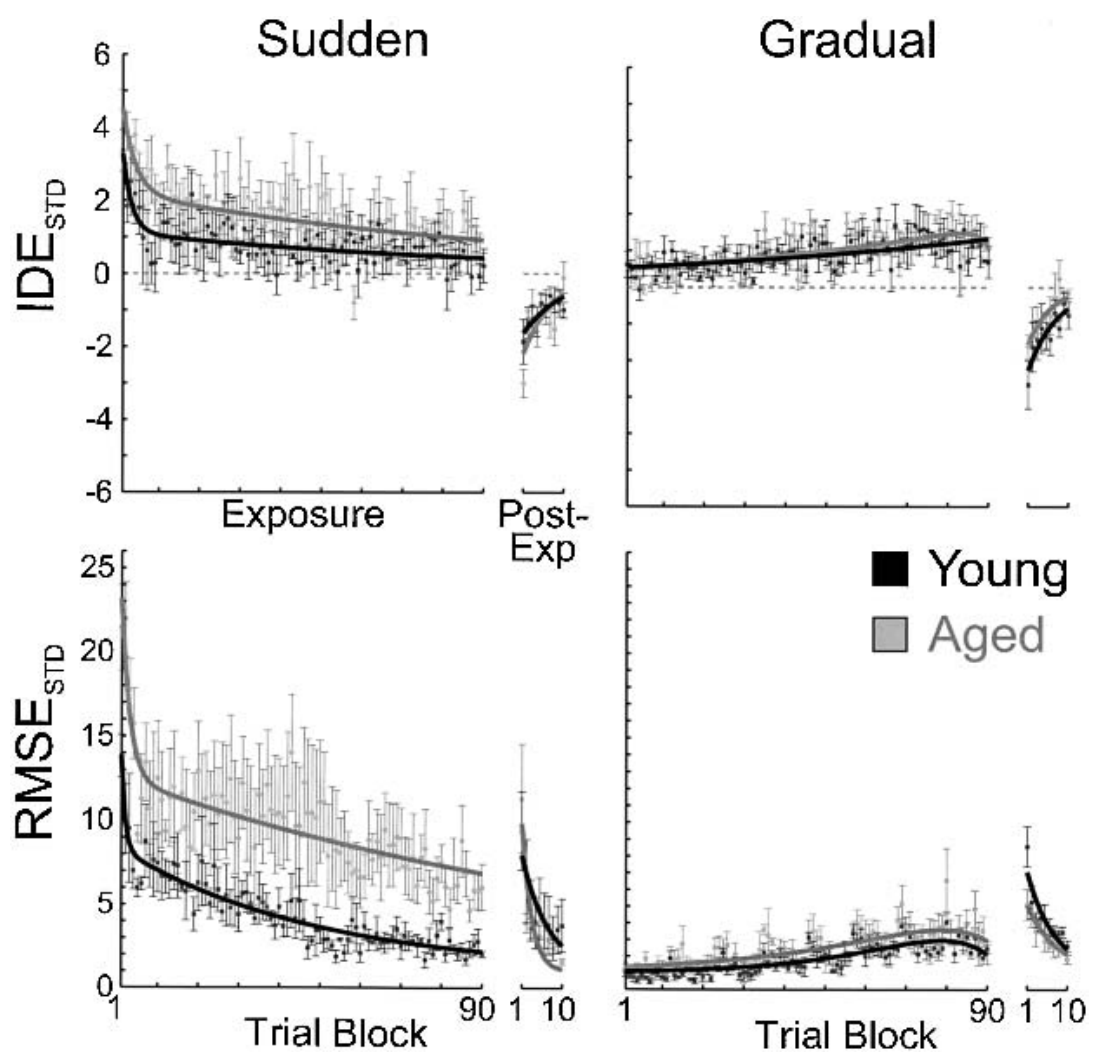

Figure 3 Trial block means, standard errors, and fitted curves for the standardized initial directional error (IDE) and root mean square error (RMSE) scores during exposure and post-exposure conditions as a function of age group and distortion type. The group data for each measure has been subdivided into two sections and fit separating the exposure (double exponential fit), and post-exposure (single exponential fit) conditions as stated by the horizontal axis label (left).

adaptation was found for standardized RMSE scores $\left(\chi^{2}=4.811, \mathrm{df}=1, P<0.05\right)$. However, whereas Figure 3 shows a trend toward a larger mean standardized IDE score in the aged group, this did not approach statistical significance $\left(\chi^{2}=0.884\right.$, $\left.\mathrm{df}=1, P=0.347\right)$, perhaps due to the large directional variability. An interesting finding is that both age groups reached the same level of adaptation by late-exposure in the gradual distortion condition $(P>0.05$ for both standardized IDE and RMSE scores), suggesting a beneficial effect of shaping by gradual exposure to the distortion.

\section{Aftereffects}

During the post-exposure trials, the aftereffect was manifested as a negative standardized IDE score (in fact in the opposite direction as that seen during early adaptation trials) and as an increase in standardized RMSE scores. Post-exposure trial-block means depicted in Figure
3 showed the typical rapid exponential return to pre-exposure levels.

Neither the first aftereffect nor the mean of the first four aftereffects revealed statistically significant differences across age groups by the Kruskal-Wallis test in the sudden adaptation task (standardized IDE and RMSE scores, both $P>$ $0.05)$. Surprisingly, in the gradual adaptation task (see Fig. 4), there were statistically significant age effects for both standardized IDE and RMSE scores when comparing the first aftereffect trial (IDE score, $\chi^{2}=3.938$, df $=1, P<0.05$; RMSE score, $\left(\chi^{2}=4.811\right.$, df $\left.=1, P<0.5\right)$. The age-related differences in post-exposure trials disappeared when the first four trials after removal of distortion were utilized for the IDE score $\left(\chi^{2}=0.884\right.$, df $=1, P>0.05)$, whereas the RMSE score showed only a marginal reduction $\left(\chi^{2}=3.153, \mathrm{df}=1, P=0.076\right)$.

\section{DISCUSSION}

The present study investigated the effects of age in visuomotor adaptation to gradual as compared with sudden screen cursor rotation. The results obtained in the present study confirm that this task is a powerful, sensitive, and reliable tool for investigating the effects of aging on the transformation, integration, modification, and storage of visuospatial and kinesthetic information for movement planning and execution. This adaptation task can be analyzed as having four major component processes. (1) The perceptual coding of the apparent location of the target and the end-effector using visuospatial and kinesthetic information, respectively; (2) the explicit selection of corrective actions (e.g., spiral,
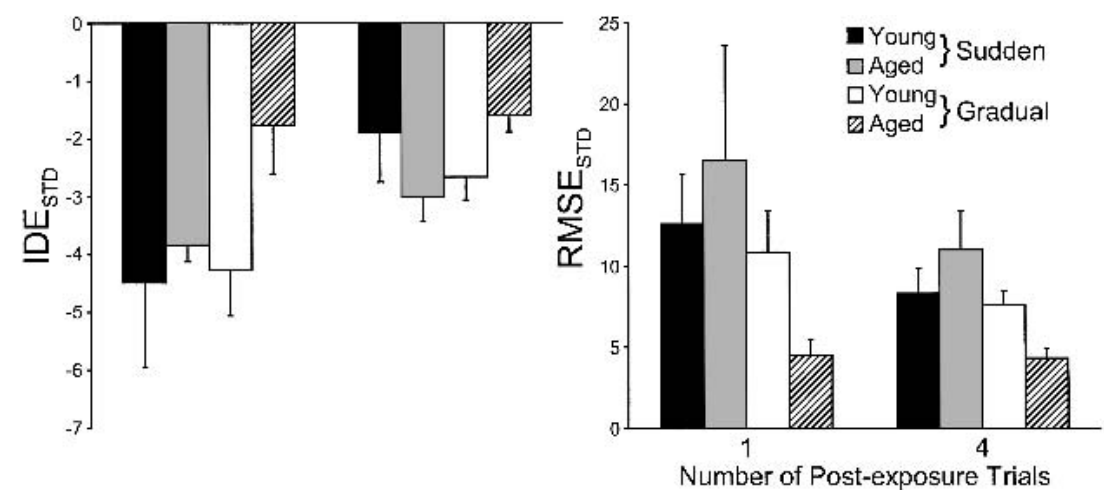

Figure 4 Aftereffect (mean and SEM) for standardized IDE and RMSE scores for the first aftereffect trial and the first aftereffect trial-block mean.

$$
\begin{array}{lllllllllllllll}
L & E & A & R & N & I & N & G & \mathbf{Q} & M & E & M & O & R & Y \\
\text { www.learnmem.org } & &
\end{array}
$$


jagged,or slow progression action) in the case of a sudden rotation, or the implicit rotation of the direction of hand movement in the gradual regime to align the hand direction to the target direction; (3) the explicit or implicit comparison between the rotated hand direction and the unrotated target direction with respect to the starting position; (4) the explicit or implicit decision on whether the hand direction and target direction align to each other; and (5) the modification and/or storage of the internal representation of the visuo-motor transformation (based on step 2) to be used in the next trial.

In the present study, we found age-related differences in both final adaptation levels and aftereffects, depending on the time course of the visual distortions. Specifically, whereas both young and aged groups show similar standardized IDE and RMSE adaptation levels throughout the exposure trials to the small stepwise gradual screen cursor rotations, the aged subjects showed higher standardized RMSE scores (but similar IDE scores) in the sudden task than the controls. Moreover, when analyzing the aftereffect trials, no age-related statistical significant differences were found for the sudden adaptation task independently of whether one or four post-exposure trials were used to analyze the aftereffects. Interestingly, when analyzing the first post-exposure trial in the Gradual adaptation task, the aged group showed smaller aftereffects, even though the final adaptation levels were comparable with those of the young participants. However, the aftereffects disappeared when four post-exposure trials were analyzed in this adaptation regime. The aftereffects seen in the first post-exposure trial in the sudden condition (thought to be free of the influence of cognitive strategies) most likely represent the learning due to explicit mechanisms, whereas the aftereffects seen in the first post-exposure trial in the gradual condition (also free of the influence of strategies) would reflect implicit learning. Moreover, our results suggest that learning and performance are differentially affected by aging; implicit learning mechanisms seem to deteriorate with age during gradual adaptation, whereas implementation of cognitive strategies in the older subjects degrades with respect to that of the young during sudden adaptation.

A puzzling outcome of the experiment was that the results from the final adaptation levels in the step rotation task showed a dissociation of IDE and RMSE scores, which was not maintained for the aftereffects. This dissociation could be explained by the following: (1) the use of different strategies (e.g., the continuing use of a jagged corrective action or loops during late adaptation, as opposed to a switch to a slow progression strategy; see Roby-Brami and Burnod 1995) in the elderly, which would result in different RMSE levels, but not necessarily larger IDE scores; and/or (2) increased movement execution variability that would affect RMSE but not IDE, as the latter is computed before feedback can affect it. The lack of aftereffect differences between age groups, and the lack of dissociation between RMSE and IDE scores in the post-exposure phase suggest that both age groups used the same cognitive strategy (e.g., CCW spirals) when exposed to the baseline (known) environment.

In agreement with the studies of Kagerer et al. (1997) and Abeele and Bock (2001), when the magnitude of the visual rotation was increased in small steps, the standardized directional (IDE) and spatial (RMSE) errors remained initially low, but then increased gradually with each step. Abeele and Bock (2001) suggested that the reduced errors observed in the gradual rotation paradigm were to be expected if adaptation during one step facilitated the adaptation during the next step (e.g., carryover). The lack of age effects during exposure in this adaptation regime may be attributed to both reduced-task complexity and increased signal-to-noise ratios in the feedback signals used for learning.

It can be argued that only the first post-exposure trial is free of volitional strategic shifts (Fernandez-Ruiz et al. 2000), and thus, is a truer indicator of learning than additional post-exposure trials. The reduced aftereffects in the aged group when comparing the first post-exposure trial may be indicative of reduced visuomotor learning under a gradual adaptation regime. Because the aftereffect differences vanished when more post-exposure trials were utilized, it is suggested that the observed deficit resulted from deterioration of implicit learning mechanisms, rather than from a decline in volitional (cognitive) strategies. Then, why is there no such effect in the sudden condition? We argue that sudden adaptation engaged explicit cognitive strategies to search for the correct visuomotor relationship, and that although the type (or mixture) of strategies may have varied across the age groups, the resulting amount of learning was similar across groups by late exposure.

\section{Comparison to Previous Studies of Visuomotor Adaptation}

Results from previous studies on visuomotor adaptation in aging are not consistent (see Table I). In the Teulings et al. (2002) experiment, the gain between the amplitude of movements of a hand-held pen and the real-time visual feedback of the vertical pen movement displayed in a displaydigitizer was either less $(70 \%)$ or greater $(140 \%)$ than normal (i.e., 1.0) in some trials (a display gain paradigm). Thus, subjects had to write larger-than-normal to compensate for the reduced display gain, or smaller-than-normal to compensate for the enlarged display gain. As expected, young subjects adapted quickly to the distorted display gain. However, the elderly subjects showed reduced adaptation and reduced aftereffects when compared with the young group. As the display gain affected progressively the subject's ongoing writing trace, this experiment could be considered a gradual adaptation paradigm. Conversely, in the prism ad-

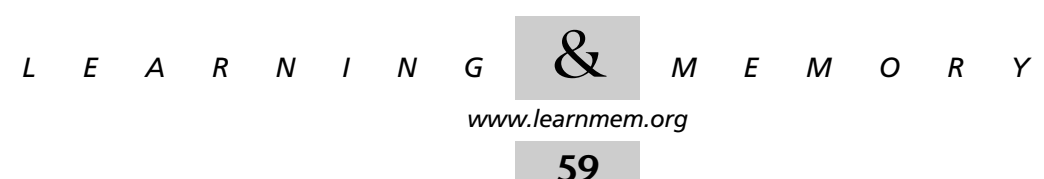


Table 1. Summary of Effects of Aging on Visuomotor Adaptation

\begin{tabular}{|c|c|c|c|}
\hline Reference & Task & Adaptation & Aftereffects \\
\hline Teulings et al. (2002) & Display gain (direct feedback) & $\mathrm{Y}>\mathrm{E}$ & $\mathrm{Y}>\mathrm{E}^{\mathrm{b}}$ \\
\hline Fernandez-Ruiz et al. (2000) & $\begin{array}{l}\text { Prism adaptation: rightward }\left(30^{\circ}\right) \text { throw on } 10 \times \\
10 \mathrm{~cm}^{2} \text { target, } 2 \mathrm{~m} \text { away }\end{array}$ & $\mathrm{Y}>\mathrm{E}$ & $\mathrm{Y}<\mathrm{E}^{\mathrm{a}}$ \\
\hline Buch et al. (this study) & Sudden display rotation $\left(\mathrm{CCW} 90^{\circ}\right)$ & $\mathrm{Y}>\mathrm{E}$ & $Y=E^{a, b}$ \\
\hline McNay and Willingham (1998) & Display rotation $\left(\mathrm{CCW} 90^{\circ}\right)$ & $\mathrm{Y}>\mathrm{E}$ & $Y=E^{*, b}$ \\
\hline Etnier and Landers (1998) & Pursuit rotor & $\mathrm{Y}>\mathrm{E}$ & N/A \\
\hline Raz et al. (2000) & Pursuit rotor & $Y>E$ & $\mathrm{~N} / \mathrm{A}$ \\
\hline Canavan et al. (1990) & $\begin{array}{l}\text { Prism adaptation: rightward }\left(30^{\circ}\right) \text { touch on target } \\
\text { arrow, } 34 \mathrm{~cm} \text { away }\end{array}$ & $\mathrm{Y}=\mathrm{E}$ & - \\
\hline Buch et al. (this study) & Gradual display rotation (up to $\mathrm{CCW} 90^{\circ}$ ) & $\mathrm{Y}=\mathrm{E}$ & $\begin{array}{l}Y>E^{a} \\
Y=E^{b}\end{array}$ \\
\hline Roller et al. (2002) & $\begin{array}{l}\text { Prism adaptation: rightward }\left(20^{\circ}\right) \text { throw on } 2 \times 2 \\
\mathrm{~cm}^{2} \text { target, } 2 \mathrm{~m} \text { away }\end{array}$ & $\mathrm{Y}=\mathrm{E}$ & $\mathrm{Y}=\mathrm{E}^{\mathrm{b}}$ \\
\hline Contreras-Vidal et al. (2002) & Display gain (indirect feedback) & $\mathrm{Y}=\mathrm{E}$ & $Y=E^{b}$ \\
\hline
\end{tabular}

$\left.{ }^{*}\right)$ Subjects were told these trials were undistorted, $(-)$ no aftereffects were measured and/or reported.

(a)Based on the first aftereffect trial only.

(b)Based on more than one aftereffect trial. Note that most studies that analyzed more than one aftereffect trial did not find significant differences across age groups. This was also the case in the present study.

aptation task of Fernandez-Ruiz et al. (2000), the elderly subjects showed slower adaptation than the control group, but after removing the prisms, the aged group showed a larger aftereffect. This result is not consistent with other reports of visuomotor adaptation in aging. Canavan et al. (1990) reported no effects of age, with no differences in number of trials to criterion, total distance incurred in misreaches or the distance of the first mis-reach during exposure between age groups. Roller et al. (2002) and ContrerasVidal et al. (2002) also reported no differences in measures of visuomotor plasticity with advancing age. Moreover, McNay and Willingham (1998), using a $90^{\circ} \mathrm{CCW}$ display rotation paradigm during line tracing, reported that elderly participants had impaired adaptation during exposure, but normal aftereffects as in the present study.

The discrepancies in prior studies may be related to task or methodological differences. Thus, in the prism adaptation studies, adaptation may require additional recalibration of the visual system with respect to the neck or trunk position, as the visual targets and visual field do not remain constant through the task (cf., Ingram et al. 2000). Moreover, the elderly populations used in the above studies may not have been homogenous. In the study by Canavan and colleagues, subjects performed verbal and spatial IQ tests (but scores were not reported), whereas in the Fernandez-Ruiz et al. study, subjects were screened for psychological disorders (but the specific test and scores were not reported either). Furthermore, in Teulings et al. (2002) and Roller et al. (2002), no neuropsychological testing was utilized in screening the elderly population. Finally, the content and level of detail in the task instructions varied widely across the studies; thus, the cognitive demands of the tasks may have varied as well. For example, in the McNay and Willingham' (1998) study, subjects were told that during post-exposure, the transformation had now been removed and they should trace the line exactly as they had in a baseline condition. These instructions may have decreased the use of volitional strategies, therefore, reducing the cognitive load in the task. Similarly, Roller et al. (2002) informed participants of the potential for visual alteration by prisms, which may account for their reported lack of age effects on adaptation.

The present results imply differential effects of aging on adaptation to gradual compared with sudden visuomotor distortions. As the participants had the same cognitive status, were told the same instructions, and performed under the same experimental apparatus, it seems from the task analysis discussed at the beginning of the Discussion, that one of the main differences between sudden and gradual adaptation is the explicit versus implicit nature of the two adaptation regimes. This, in turn, translates into differences in the awareness of the large versus small visual distortions, and in the selection and use of cognitive strategies versus implicit learning mechanisms. The second difference relates to the level of task complexity (e.g., amount of visual distortion), which in turn had an influence on the level of spatiotemporal variability of the response (cf., for example, the error bars in gradual vs. step rotation in Fig. 3). Furthermore, the magnitude in the aftereffects levels in gradual adaptation depended on whether one or more post-exposure trials were included in the analysis. This suggests that when cognitive strategies are removed from the task (as is the case of the first post-exposure trial), aging effects may become significant if explicit cognitive strategies were not used for learning. Thus, part of the conflicting results summarized in Table 1 may be accounted for by the number of post-exposure trials used to assess visuomotor learning.

Current behavioral theories suggest that cognitive de-

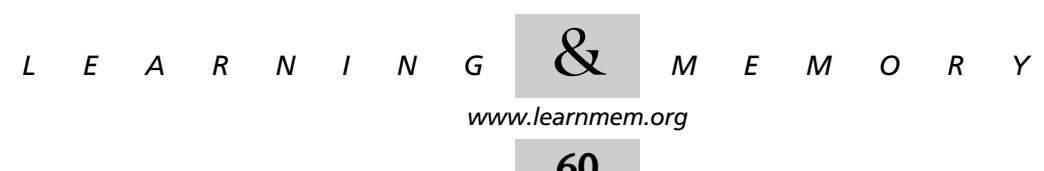


cline with aging is due to decreases in cognitive resources, such as verbal and visuospatial working memory, ability to switch among tasks, inhibitory function, and speed of processing (Salthouse 1996; Park et al. 2002). Usually, cognitive tasks that require effortful processing are thought to require working memory, and are the ones that show the greatest detrimental effect of aging. Conversely, recent studies suggest that memory decline does not occur on tasks with low executive needs (i.e., little effortful and strategic processing), such as in implicit memory tasks (Park and Shaw 1992). In our adaptation task, we found that visuomotor learning in the explicit, effortful, strategic task (sudden rotation) was equivalent across age groups, although performance was deteriorated for the elderly. However, performance in the implicit, nonstrategic task (gradual rotation) was similar across age groups, but visuomotor learning was reduced in the elderly (as measured by the one-trial aftereffects). This is contrary to recent findings of negative effects of aging on associative memory tasks in the language domain (i.e., learning of novel word-pair associations) that rely on consciously controlled process as opposed to automatic, unconscious processes (O'Hanlon et al. 2001). The present study suggests that aging may differentially affect automatic versus conscious controlled processes in visuospatial tasks. However, more experiments engaging visuospatial processes are needed to confirm this view.

Our main finding of differential effects of aging on visuomotor adaptation to gradual and sudden distortions may have clinical implications. In terms of performance, gradual adaptation resulted in less directional and spatial errors, but reduced aftereffects, whereas step adaptation resulted in normal aftereffects, but increased spatial variability during adaptation. In clinical settings, shaping procedures, such as the gradual distortion herein, are used to gradually train patients after stroke. The present results support the use of this therapeutic strategy as a means of enhancing visuomotor adaptation to altered conditions in the normal aging population, as adaptation levels across exposure were identical for young and aged participants. However, the reduced aftereffects observed indicate that shaping may not entirely circumvent visuomotor learning deficits associated with normal aging.

In summary, these findings suggest that in terms of visuo-motor performance during sudden adaptation, the older subjects are not able to implement cognitive strategies as effectively as the young participants; nevertheless they were able to adapt to the same extent as evidenced by the similar levels of aftereffects across age groups. In the case of the gradual regime, which does not involve explicit corrective actions, the performance during exposure is comparable across age groups; however the smaller aftereffects in the older subjects indicate that mechanisms for implicit learning, which presumably were minimally engaged during the sudden condition, are deteriorated in this age group.

\section{METHODS}

\section{Subjects}

Ten healthy elderly participants between the ages of 63 and 80 (five males and five females; mean age of $73.3 \pm 6.70 \mathrm{yr}$ ) and ten healthy young controls between the ages of 18 and 25 (five males and five females; mean age of $21.00 \pm 2.70 \mathrm{yr}$ ) were recruited from the local university and from advertisements. Both women and minorities were represented in the groups. The subjects were naive to the purpose of the experiment, and all subjects were right-handed. All subjects had normal or corrected-to-normal vision, gave informed written consent, and answered a health-history questionnaire. All participants were given the temporal orientation, registration, attention and calculation, recall, and language items of the MiniMental State Examination test (elderly, MMSE score of $23.7 \pm 1.9$; young, MMSE score of $24.8 \pm 0.6$; Folstein et al. 1975). Elderly participants were paid for their participation, whereas young controls received class credit. All procedures were approved by the Institutional Review Board at the University of Maryland at College Park.

\section{Apparatus}

Each participant sat at a table facing a computer screen $(41 \times 30 \mathrm{~cm})$ in front of him/her at a distance of $60 \mathrm{~cm}$ (Fig. 1A). A vertical board was placed on the table between the subject's head and right shoulder to occlude vision of the arm and hand. An infrared (IRED) marker was attached to the tip of the right index finger. The position of this marker was sampled in real time at 100 $\mathrm{Hz}$ via an infrared three-dimensional motion measurement system (Northern Digital Optotrack) connected to a personal computer (Gateway 2000 E-4200). Feedback of the tip of the (extended) index finger position was presented in the form of a white screen cursor (5-mm diameter). The subjects controlled the movement of the screen cursor by sliding the wrist and forearm across the surface of the table to the right of the occluding board. This effectively constrained the movement to the horizontal plane. Subjects were instructed to make point-to-point movements "as fast and as straight as possible when ready" by moving the white screen cursor from a common central starting (home) location to one of four (pseudo-randomized) target circles (diameter, $10 \mathrm{~mm}$; directions, $45,135,225$, and $315^{\circ}$ ) displayed on the screen at $20 \mathrm{~cm}$ from the home circle. The start position and all four targets were visible throughout the entire duration of the testing session. Movements were initiated following the presentation of an auditory GO (400$\mathrm{Hz}$ tone) signal. Brief rest periods were allowed as needed to minimize fatigue and maintain attention.

\section{Experimental Procedure}

Both age groups were equally divided at random into two subgroups; one subgroup was exposed to $11.25^{\circ} \mathrm{CCW}$ step increments, every 45 trials, of visual feedback rotation up to a total of $90^{\circ}$ (gradual rotation), whereas a second subgroup was subjected to $90^{\circ} \mathrm{CCW}$ cursor rotation from the onset of exposure (sudden rotation; see Fig. 1B). The experimental session consisted of 3 conditions that covered 440 total trials. During the pre-exposure condition (40 trials; 10 movements per target direction), subjects moved to one of four targets in the absence of any visual feedback distortion. During the exposure condition, trials 41-400 (360 trials; 90 movements per target direction) were performed under a gradual or sudden rotation applied to the screen cursor representation of the index finger position. Finally, trials 401-440 (40 trials;

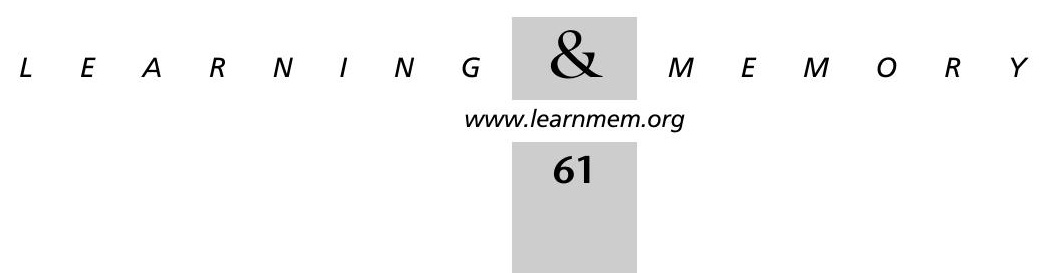


10 per target direction) were performed with normal visual feedback to test for aftereffects (post-exposure condition). The target directions were randomized within each condition and consistent between all subjects. Data acquisition was initiated with the acoustic start signal and terminated once the screen cursor reached the prespecified target. Subjects had a maximum of $10 \mathrm{sec}$ in which to complete the trial. If this time constraint was reached, subjects were instructed to immediately terminate the current movement and return to the home position, where they awaited commencement of the subsequent trial. Subjects were allowed a few practice trials (with normal visual feedback) before testing began to familiarize them with the experimental setup.

\section{Screen Cursor Rotation Transformation Rule}

During the exposure condition, the subjects controlled the position of the cursor $(x, y)$ which corresponded to the position of the actual index finger $(p, q)$ rotated about the center of the work space:

$$
\left(\begin{array}{l}
x \\
y
\end{array}\right)=\mathbf{R}\left(\begin{array}{l}
p \\
q
\end{array}\right)=\left(\begin{array}{cc}
\cos \theta & -\sin \theta \\
\sin \theta & \cos \theta
\end{array}\right)\left(\begin{array}{l}
p \\
q
\end{array}\right)
$$

The rotation matrix $\mathbf{R}$ was used with $\theta=90^{\circ}$ in the Sudden Condition, whereas in the Gradual Condition $\theta$ was increased by $11.25^{\circ}$ every 45 trials during the exposure stage (the sequence of $\theta$ 's was given by $11.25,22.5,33.75,45,56.25,67.5,78.75$, and $90^{\circ}$ ).

\section{Data Acquisition and Analysis}

To reduce noise in the signal due to quantization and jittering (high frequency changes) of the infrared signal, the sampled Cartesian position data were low-pass filtered using an 8th order Butterworth filter with a high cut-off of $5 \mathrm{~Hz}$. Dual-pass filtering was used to eliminate phase distortion. The smoothed data allowed the numerical differentiation of the tangential position, which was used to obtain the velocity time series for each movement. Movement onset was determined by finding the velocity zero crossing immediately preceding the first point in the velocity time series that was at least $20 \%$ of the peak velocity. A vector from the initial (home) hand position to the position of the hand at $80 \mathrm{msec}$ after movement onset, determined the initial direction of the planned movement trajectory. The initial directional error (IDE, in degrees) was calculated as the angular difference between this vector and a vector extending from the starting (home) position to the target. Assessment of the directional error $80 \mathrm{msec}$ after the movement onset allows the directional error to be measured before corrections guided by visual feedback are used. IDE can be considered to represent the planning of the initial direction of movement, and thus, the current state of the internal model of the rotated environment.

The root mean square error (RMSE, in millimeters) was calculated to assess the average deviation of the movement trajectory from a straight line connecting the starting point of the movement and target position as follows,

$$
\operatorname{RMSE}(\text { in } m m)=\sqrt{\frac{1}{N} \sum_{i=1}^{N}\left(x_{a}-x_{i}\right)^{2}+\left(y_{a}-y_{i}\right)^{2}}
$$

in which $\left(x_{a}, y_{a}\right)$ and $\left(x_{i}, y_{\mathrm{i}}\right)$ are corresponding points of the spatially resampled (to achieve equally spaced samples) movement trajectory and the ideal trajectory (whose temporal structure was characterized by a uniform velocity profile and equidistant samples), respectively, and $N$ is the number of points in the trajectory. Using the resampled hand trajectories, the mean $( \pm \mathrm{SD})$ hand paths were computed and plotted for pre-, early, late-, and post- exposure trials. As the RMSE score is computed along the hand trajectory from the home to the target, it can be seen as comprising both planning and execution (due to implementation of planned motor commands and on-line feedback-based corrections, respectively) components of adaptation. Moreover, the RMSE scores would depend on the particular strategy used to deal with the task requirements. Roby-Brami and Burnod (1995) showed that the corrective actions during exposure to visual rotation were a combination of three types of movement trajectories, spiral, angle or jagged, and slow progression, with spiral trajectories almost always produced during the early exposure trials. Most likely, spiral trajectories would produce the largest RMSE scores, whereas slow progression movements, which are irregularly curved but usually in the correct direction, would produce the smallest RMSE. Thus, it is possible that IDE and RMSE scores during adaptation may diverge, depending on the correction strategy used and the degree of reliance on visual feedback, and they can be considered to capture different aspects of adaptation.

To take into account baseline differences due to increased spatio-temporal movement variability with advancing age (Cooke et al. 1989; Darling et al. 1989; Contreras-Vidal et al. 1998; Teulings et al. 2002), standardized scores were computed from the IDE and RMSE variables. The standardized score (z-score) for an item indicates how far and in what direction that item deviates from its distribution's mean, expressed in units of its distribution's standard deviation. This transformation is also useful to compare the relative magnitudes of IDE and RMSE scores, which are known to come from distributions with different means and different standard deviations (Pine et al. 1996). Moreover, standardization of the error scores allowed us to focus on the changes in IDE and RMSE due to adaptation with respect to baseline performance within each group. For each subject, the standardized scores for each item (IDE, RMSE) were obtained using the equation,

$$
z_{i}=\frac{\left(x_{i}-\bar{x}_{\text {Pre-exposure }}\right)}{S D_{\text {Pre-exposure }}}
$$

$x_{i}$ is the score for a single exposure or post-exposure trial, $\bar{x}_{\text {Pre-exposure }}$ is the pre-exposure mean of the measure, $S D_{\text {Pre-exposure }}$ is the preexposure standard deviation, and $z_{i}$ is the corresponding standardized score. Measurements from four consecutive trials were pooled and one block mean was calculated for IDE and RMSE. Both double and single exponential curves were used to fit the group data for the exposure and post-exposure conditions (using the Nelder-Mead Simplex Algorithm as implemented in the MATLAB function fminsearch), and plotted as a function of trial block. The residual errors (root mean square errors and the residuals normalized to the mean score) were used to assess goodness of fit and select the number of parameters in the fitting equations. To avoid overfitting, a penalty factor $([\mathrm{N}+\mathrm{P}] /[\mathrm{N}-\mathrm{P}]$, in which $\mathrm{N}$ is the number of samples, and $\mathrm{P}$ is the number of parameters) that increases with the number of parameters was used to weight the modeling error. This criterion is similar to the final prediction error (FPE; Manolakis et al. 2000) used in parametric signal modeling. Polar plots were also used to show the rotation of the mean $( \pm \mathrm{SD})$ initial movement direction relative to pre-exposure as a function of learning stage.

A multivariate analysis of variance using the between-subject factors group (Young; Elderly) and the within-subject factor rotation angle for IDE and RMSE was obtained to assess effects of group, effects of rotation angle, and group $\mathrm{x}$ rotation interaction during adaptation to a gradual screen cursor rotation. KruskallWallis nonparametric statistical tests were used to assess initial

\section{$\begin{array}{lllllllllllllll} & E & A & R & N & I & N & G & \mathcal{Q} & M & E & M & O & R & Y \\ \text { www.learnmem.org } & & & \end{array}$}


response (early-exposure) to the distorted environment, final adaptation levels (late-exposure), and post-exposure trials (aftereffects) as in Roller et al. (2002). Both the first available aftereffect trial and the first trial-block (mean of first four post-exposure trials) were analyzed. Analysis of the first aftereffect trial allowed us to minimize volitional cognitive strategies and to account for the transient nature of the aftereffects, which is typical of paradigms that use representational feedback of hand movement (Kagerer et al. 1997; Clower and Boussaoud 2000).

\section{ACKNOWLEDGMENTS}

Supported by NIA grant AG19148 and the Howard Hughes Medical Program.

The publication costs of this article were defrayed in part by payment of page charges. This article must therefore be hereby marked "advertisement" in accordance with 18 USC section 1734 solely to indicate this fact.

\section{REFERENCES}

Abeele, S. and Bock, O. 2001. Sensorimotor adaptation to rotated visual input: Different mechanisms for small versus large rotations. Exp. Brain. Res. 140: 407-410.

Canavan, A.G., Passingham, R.E., Marsden, C.D., Quinn, N., Wyke, M., and Polkey, C.E. 1990. Prism adaptation and other tasks involving spatial abilities in patients with Parkinson's disease, patients with frontal lobe lesions and patients with unilateral temporal lobectomies. Neuropsychologia 28: 969-984.

Clower, D.M. and Boussaoud, D. 2000. Selective use of perceptual recalibration versus visuomotor skill acquisition. J. Neurophysiol. 84: $2703-2708$.

Contreras-Vidal, J.L., Teulings, H.L., and Stelmach, G.E. 1998. Elderly subjects are impaired in spatial coordination in fine motor control. Acta Psychologica 100: 25-35.

Contreras-Vidal, J.L., Teulings, H.L., Stelmach, G.E., and Adler, C.H. 2002. Adaptation to changes in vertical display gain during handwriting in Parkinson's disease patients, elderly and young controls. Parkinsonism Rel. Disord. 9: 77-84.

Cooke, J.D., Brown, S.H., and Cunningham, D.A. 1989. Kinematics of arm movements in elderly humans. Neurobiol. Aging 10: 159-165.

Darling, W.G., Cooke, J.D., and Brown, S.H. 1989. Control of simple arm movements in elderly humans. Neurobiol. Aging 10: 149-157.

Etnier, J.L. and Landers, D.M. 1998. Motor performance and motor learning as a function of age and fitness. Res. Q. Exerc. Sport. 69: $136-146$

Fernandez-Ruiz, J., Hall, C., Vergara, P., and Diaz, R. 2000. Prism adaptation in normal aging: Slower adaptation rate and larger aftereffect. Brain Res. Cogn. Brain. Res. 9: 223-226.

Folstein, M.F., Folstein, S.E., and McHugh, P.R. 1975. "Mini-mental state". A practical method for grading the cognitive state of patients for the clinician. J. Psychiatr. Res. 12: 189-198.

Ingram, H.A., van Donkelaar, P., Cole, J., Vercher, J.L., Gauthier, G.M., and
Miall, R.C. 2000. The role of proprioception and attention in a visuomotor adaptation task. Exp. Brain Res. 132: 114-126.

Kagerer, F.A., Contreras-Vidal, J.L., and Stelmach, G.E. 1997. Adaptation to gradual as compared with sudden visuomotor distortions. Exp. Brain Res. 115: 557-561.

Manolakis, D.G., Ingle, V.K., and Kogon, S. 2000. Statistical and Adaptive Signal Processing: Spectral estimation, signal modeling, adaptive filtering, and array processing. McGraw Hill, New York., NY.

McNay, E.C. and Willingham, D.B. 1998. Deficit in learning of a motor skill requiring strategy, but not of perceptuomotor recalibration, with aging. Learn. Mem. 4: 411-420.

Mirmiran, M., van Someren, E.J., and Swaab, D.F. 1996. Is brain plasticity preserved during aging and in Alzheimer's disease? Behav. Brain Res. 78: $43-48$.

O'Hanlon, L., Wilcox, K.A., and Kemper, S. 2001. Age differences in implicit and explicit associative memory: Exploring elaborative processing effects. Exp. Aging Res. 27: 341-359.

Park, D.C. and Shaw, R.J. 1992. Effect of environmental support on implicit and explicit memory in younger and older adults. Psychol. Aging 7: 632-642.

Park, D.C., Lautenschlager, G, Hedden, T., Davidson, N.S., Smith, A.D., and Smith, P.K. 2002. Models of visuospatial and verbal memory across the adult life span. Psychol. Aging 17: 299-320.

Pine, Z.M., Krakauer, J.G., and Ghez, C. 1996. Learning of scaling factors and reference axes for reaching movements. Neuroreport 7: 2357-2361.

Pratt, J., Chasteen, A.L., and Abrams, R.A. 1994. Rapid aimed limb movements: Age differences and practice effects in component submovements. Psychol. Aging 9: 325-334.

Raz, N., Williamson, A., Gunning-Dixon, F., Head, D., and Acker, J.D. 2000. Neuroanatomical and cognitive correlates of adult age differences in acquisition of a perceptual-motor skill. Microsc. Res. Tech. 51: 85-93.

Richardson, J.T.E. and Vecchi, T. 2002. A jigsaw-puzzle imagery task for assessing active visuospatial processes in old and young people. Behavior Res. Methods, Instruments Computers 34: 69-82.

Robertson, E.M. and Miall, R.C. 1999. Visuomotor adaptation during inactivation of the dentate nucleus. Neuroreport 10: 1029-1034.

Roby-Brami, A. and Burnod, Y. 1995. Learning a new visuomotor transformation: error correction and generalization. Brain Res. Cogn. Brain Res. 2: 229-242.

Roller, C.A., Cohen, H.S., Kimball, K.T., and Bloomberg, J.J. 2002. Effects of normal aging on visuomotor plasticity. Neurobiol. Aging 23: $117-123$.

Salthouse, T.A. 1996. The processing-speed theory of adult age differences in cognition. Psychol. Rev. 45: 403-428.

Teulings, H.L., Contreras-Vidal, J.L., Stelmach, G.E., and Adler, C.H. 2002. Adaptation of handwriting size under distorted visual feedback in patients with Parkinson's disease and elderly and young controls. $J$. Neurol. Neurosurg. Psychiatry 72: 315-324.

West, R.L. 1996. An application of prefrontal cortex function theory to cognitive aging. Psychol. Bull. 120: 272-292.

Received May 17, 2002; accepted in revised form December 3, 2002. 


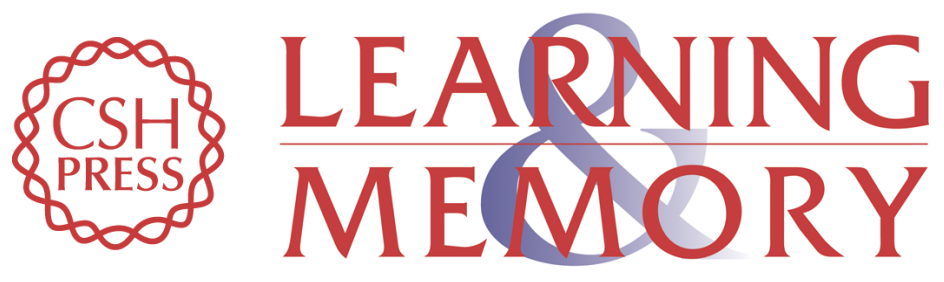

\section{Visuomotor Adaptation in Normal Aging}

Ethan R. Buch, Sereniti Young and José L. Contreras-Vidal

Learn. Mem. 2003, 10:

Access the most recent version at doi:10.1101/lm.50303

References This article cites 26 articles, 2 of which can be accessed free at: http://learnmem.cshlp.org/content/10/1/55.full.html\#ref-list-1

License

Email Alerting Receive free email alerts when new articles cite this article - sign up in the box at the Service top right corner of the article or click here. 